TM-1458

2320.000

\title{
List Processing Software for the LeCroy 1821 Segment Manager Interface*
}

\author{
T. Dorries, C. Moore, and R. Pordes \\ Data Acquisition Software Group \\ Fermi National Accelerator Laboratory \\ P.O. Box 500, Batavia, Illinois 60510
}

May 1987

*Presented at the Fifth IEEE Conference on Real-Time Computer Applications in Nuclear, Particle, and Plasma Physics, San Francisco, California, May 12-14, 1987. 
LIST PROCESSING SOFTWARE FOT THE

LECROY 1821 SEGMENT MANAGER INTERFACI

Terry Dorries. Carmenita Moorc. Ruth Pordes

Datí A coussion Sotrware Groun"

Fermi Nationa! Acceierator Laborator:

P.O. Box 500. Baravja, Il 60510

\section{Spting the Scene}

The LeCroy 1821 Segment Manager is a FASTBUS host interface that is used in experiments at Fermilab at two ievels of the event. data readout.' At the inwest level the 1821 s are emploved in frontend crates to read TDC and ADC modujes. In oome cases 1821 s operating ai this level rend the dath directly into a host computer; in other cases they store the dati in intermediate buffer memories such as the LeCroy 1892 multiple record bufier memory:

At the second level the 182 is are used in the (single) host FASTBUS crate at the apex of tine data readout. Here the $1821 \mathrm{~s}$ are used to reac data into the data acquisition computer from the bufíe memories in which many hundreds of previously gathered events have beer stored.

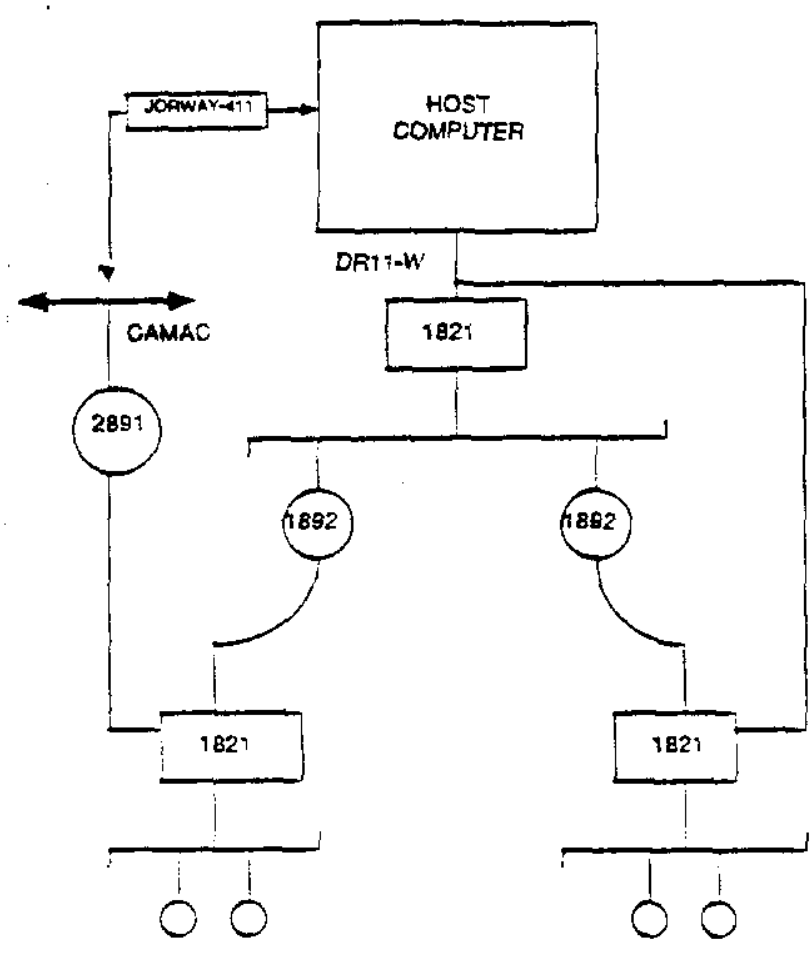

Figure 1: Topoiogy of Experiments Using 1821

The Data Acouisjion Sofrware Group in the Computing Leparmeni ai Fermilab proviaes support software for the 1821 to run on VAX/VMS. PDP. $11 / \mathrm{RSX}-11 \mathrm{M}$ and PDF-11/RT-1] systems. The softwarc has been develoned in two phases.

The first pinase provices a package of low ieve! access routines wich aliow the uset to immediateiy execute any one of many 1821 specific functions. The second phase, which is only avainable on $R S X-11 M$ and VMS systems, provides for the definition and execution of lists of 1821 opcrations. By executing issts of 1821 operations many 1821 functions can be performed in a single $1 / O$ request to the device driver thus significantly reducing the sotrware overheads associated with the use of the 1821. This paper will concentrate on the list processor implementations. Since the low level routinc packuge is used by general user level programs, it will be briefly' discussed.

\section{Connection to thr $1821 \mathrm{~s}$}

A LeCroy 2821 Segment Manager can be controlled by a host computer through one of two connection patis. The LeCroy 2891 interface Module is used to interface the 1821 to CAMAC aliowing the host computer access to the 1821 tinrough CAMAC. Alternately the 1821 can be connected to a UNIBUS or QBUS tinrough a DRII-W DMA interface." in the iatter case. if several 182 Is are chained together, at least one 1821 in the chain must be connected tinrough the auxiliary port to the DRIJ- $K$ using a LeCroy $1821 / \mathrm{DEC}$ interface cart. Other 1821 i may be chajned to this primary, either through connections to their front panel or, with a slight modification of the standard $1821 / D E C$ card, timrough a private $1621 / \mathrm{DEC}$ connected to their auxiliary port.

Note that for a host computer with a UNIBUS connection, the 1821 may be connected usinf a DEC DRld-W. When the conrection is to a QBUS, the DEC DRV1]-W has been found not to wor). (This is becauke the $1821 / \mathrm{DEC}$ relias nt the GO ktroine sio jnitjate jts operations, and the DEC DRYlu-W does not provide this stsobe to the user device.) Fine $\mathrm{MDB}{ }_{3}$ Systems MLSi-DR1;-W interface, nowever, does work. 3

Lov: Level 182] Access Routine anc' Sunnor: Programs

The low level access routines are a Fortran routine package thai provioe aset interface to the 1821. The package contains routines for reading and writing $182 \mathrm{l}$ host $1 / 0$ registers, downloading and execuring 1822 microcode programs. aownioading peoestals, and reaōing out the 1821 's internal memory.

Iwo diagnostic tools, one which tests tine internal integruty and functions of an 1821, and one winich provicies an interpretive environment for $t$ user to specify 1822 operations to be performed, have been developed based on the iow ievel routines. 4

\section{Overviev: of the Eie Peocessors}

Fo: a use:'s program to access the 1821 tic host's opcrating system mus piace ay $1 / 0$ reouest to tine 1821 acver driver. The time required for tile system to issue such recuest js or the orcicr of 2 to 4 miliseconds. Severial $182 \mathrm{j}$ functions must be done 
for the 1821 to perform any useful function. For example, using the 1821 microcode provided by LeCroy, an operation $w$ read a FASTBUS data word (if it is to follow the FASTBUS specification of an operation) will require between 4 and 6 functions performed on or by the 1821 . Clearly the software overhead imposed by accessing the 1821 on a per function basis is too restrictive in a real time data acquisition environment.

In order to minimize the number of $\mathrm{I} / \mathrm{O}$ requests issued by the system. list processing drivers have been developed. Suchi drivers exist for the RSX-11M and VMS systems for 1821 s connected through a DR11-W to the host. These drivers allow the user to perform a list of 1821 and FASTBUS operations through a single $1 / O$ request. The drivers are a significant extension to the original drivers provided by LeCroy (VMS) and Ohio State University (RSX). ${ }^{6,7}$

In addition to the device drivers, the low level Fortran access routines have been extended to ajlow building, executing. saving and interrogating the lists and the results of their execution.

\section{List Structure}

The list structure developed consists of fixed length 8 byte list elements. Each list element specifies an 1821 control opcration, DMA readout of the 1821 data memory. microcode execution, or a control or arithmetic function. The list structure is designed to ease the later implementation of the standard routines for FASTBUS ${ }^{b}$ for the 1821. The list eiement structure is compatible with the so called "F-Code" format, defined by CERN for FASTBUS lists. ${ }^{9}$ The available list element opcodes provide enough flexibility and functionality that a whole data acquisition read-out can be specified by a single, of at the most two, lists.

The list structure allows for:

- definition of control parameters to vary the options and parameters associnted with exeristion of each list eiement.

- read/write of individual $1821 \mathrm{I} / 0$ registers,

- execution of 1821 microcode routines with and without data from the host computer,

o readout of the 1821 data memory within the list.

o 3 internal variables for manipulation within the list.

o arithmetic and logical functions to be performed on the data read or written, the status returned by the 1821 , or the internal variabies,

o immediate or conditional branching within a list,

o execution of DO loops within the list,

- 16 or 32-bit mode data manipulation,

o separate read and write data buficrs,

o return of status information from the execution of the list, o return of status fron tinc execution of eacl operation within a list.

o inclusion of an 'Even: Header' in the data buffer (for the VMS implementation onjy).

\section{Timing:}

The following timings have been measured for the two list processing drivers. A $Y A X / 78(1$ computer was used for the VMS timing rests and the RSX tests were run on a PDP-1//4j. The null list times represent the time required for the user's process to invoke the device driver, the driver software to do the setup necessary for list execution, and for the driver to return control to the user's process; that is, the one time overhead per lis execution. The overhead per list element varies significantly with the actual operation being performed. The total overhead for a particular list is the sum of the per list eiement overheads for each list element within the list and tise null list overhead.

\begin{tabular}{lll} 
& \multicolumn{1}{c}{ VMS } & $\mathrm{RSX}$ \\
\hline Null list: & $6.7 \mathrm{Msec}$ & $4.1 \mathrm{Msec}$ \\
$\begin{array}{l}\text { Overhead per list } \\
\text { element: }\end{array}$ & $160-360$ usec & $400-850 \mathrm{usec}$ \\
$\begin{array}{l}\text { Overhead for single } \\
\text { operation using non- } \\
\text { list processing } \\
\text { device driver: }\end{array}$ & $4 \mathrm{Msec}$ & $2 \mathrm{Mrec}$ \\
\hline
\end{tabular}

\section{Jiser Subroutine Library}

The Fortran subroutine library provided allows a use program to dynamically build and execute iists. Subromitines are availabic to constinet list elements, execute, save or restore a list, change options and parameters associated with list execution, and interrogate the status and data returned from execution of the list. 10

\section{List Generation and Execution Tools}

Programs have been developed fo: both RSX-11M and VMS systems to provide intcractive features for performing list operations through the 1821 . These programs allow the user to interactively define, edit and execute the 1821 lists. The programs can either create lists dynamicaliy or read in "source codc" list files (ASCII text files containing list ejement definitions). Once a lisi has becr created or read in from a disk file it is compiied into an executable form. The compiled lists can then be executed and/or saved on disk for later execution. If the uker chooses to execute the list then the programs car be used to interrogate the results - both data and status information. 11.12 


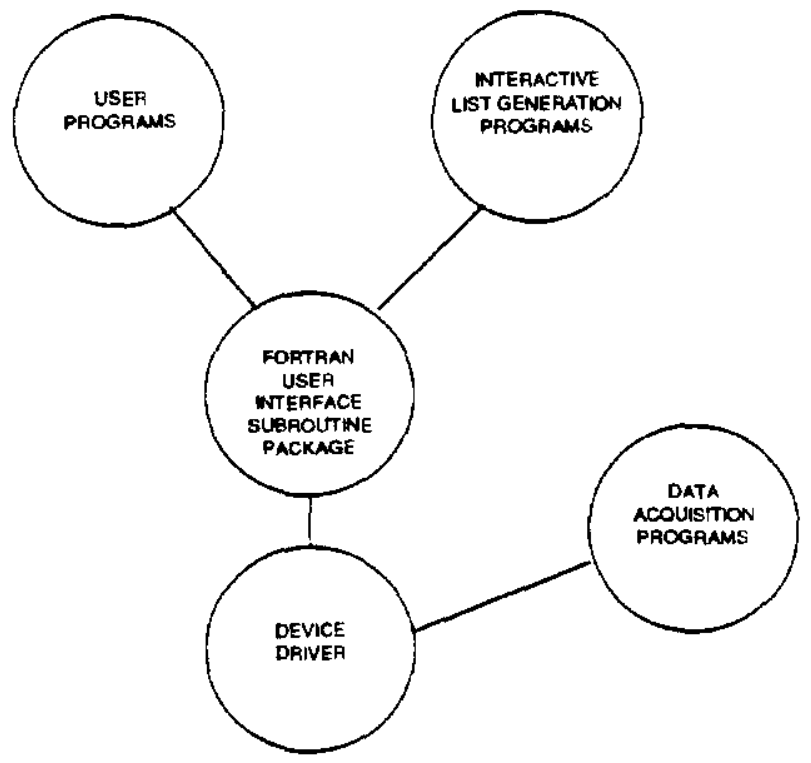

Figure 2: Overviet of the 1821 Soltwarc

Datí Acouisition Applications

Execution of 1821 lists on receipt of an event interrupt has been incorporated into the Fermilab standard RSX-11M data acquisition program $^{13}$ and is in use at two experiments. Data acquisition through the VMS $182 \mathrm{l}$ list processing driver has been incorporated into the VAXONLINE Event Builder, ${ }^{14}$ and will be used in a fixed target experiment during the upcoming run. It is interesting to note that this last experiment uses a list of some 100 operations to provide sophisticated control of which modules should be read out, and what $182 \mathrm{I}$ microcode routines are to be executed.

In both data acquisition systems, the 1821 lists are constructed 'offline' using the list generation tools. They may be executed for test purposes using these tools, and then saved on disk for later execution by the data acquisition programs themselves.

\section{Summary}

Many fixed target experiments at Fermilab now include some FASTBUS electronics in their data readout. The software reported in this paper, provides general support for the LeCroy 1821 interface. The list processing device drivers allow FASTBUS data to be read out efficiently into the Fermilab Computing Department supported data acquisition systems.

\section{Acknowledgments}

As usual many people have contributed to the work reported in this paper. All members of the Data Acquisition Software group contribute to any project being developed in its midst. For the projects reported in this paper, in particular. many experimenters at Fermilab have contributed to the detailed understanding of tine LeCroy harowarc, and the testing of software for the 1822 .
1. LeCroy Corporation, 700 so. Main Strect. New lork 1097.

2. DR11-W Direct Memory Interface Modulc, Digital Equipment Corporation

3. MDB Systems. Inc., Orange, CA 92605.

4. T1821. Lecroy-1821 Diagnostic Program, Fermilab, PBN-040.

5. LeCroy 1821 SM/I Diagnostic Tool (LDT), Fermilab, FBN-(1)1.

6. NYCB Computing, Inc., Lecroy 1821 Device Driver.

7. John W. Heimaster, Ohio State University.

8. Proposed IEEE Standard Routines for FASTDUS FASTBUS Software Working Group, February 198\%, Fermilab) FBN-051.

9. CERN's Approach to FASTBUS Software. E.M Rimmer, IEEE Transactions on Nuclear Science, 3968, Vol. NS-30, October 1983.

10. Low Level Routines for the Lecroy 1821 , Fermilab FBN-039.

11. FLIST, An Interactive Program for Generating Lecroy 1821 Lists for RSX-11M, Fermilab FBN0048.

12. Interactive List Generation Program FLMENC, Fermilab FBN-0047.

13. A High Speed CAMAC Data Acquisition System for PDP-11, D.M. Berg. et al. IEEE Transactions on Nuclear Sciencc, 1368, Vol. NS32.

14. Sec companion papet to this conference, The VAXONLINE Software System at Fermilab, $V$. White, et al. 